\title{
CHARLES DARWIN: INMORTALIZADO EN EPÓNIMOS DE GEA, FLORA Y FAUNA RECIENTES DE CHILE ${ }^{1}$
}

\author{
CHARLES DARWIN: INMORTALIZED IN EPONYMS OF GEA \\ AND RECENT FLORA AND FAUNA OF CHILE
}

Vicente Pérez ${ }^{2}$

\section{RESUMEN}

Se presentan epónimos de gea, flora y fauna de Chile, erigidos en honor de Charles Darwin, naturalista a bordo del H.M.S. Beagle (1831-1836), para denominar accidentes geográficos de territorio chileno y nuevas especies de flora y fauna descritas de ejemplares recolectados en Chile por él.

Palabras clave: Beagle, Darwin, angiospermas, insectos, pteridófitas, vertebrados.

\section{ABSTRACT}

Eponyms of gea, flora and fauna, erected in honour of Charles Darwin, naturalist on board of the H.M.S. Beagle (1831-1836), for designate geographic accidents and new species of flora and fauna described from specimens collected by him in Chile.

Key words: Beagle, Darwin, angiospermae, insects, pteridophytes, vertebrates.

\section{INTRODUCCIÓN}

Las expediciones han sido las principales generadoras de topónimos de las regiones que han descubierto y explorado; al mismo tiempo, la recolección de ejemplares de flora y fauna proporcionó el material que permitió a los taxónomos describir nuevas especies para la ciencia, lo cual agradecieron erigiendo epónimos en honor de los recolectores. Esto último se ha convertido en una práctica constante hasta nuestros días, ya que ha sido una manera de estimular la labor de los recolectores, sean éstos destacados hombres de ciencia, aficionados amantes de la naturaleza que obsequian los ejemplares a los especialistas o comerciantes especializados en este rubro. Sean lo que fueran, siempre sale beneficiada la ciencia.

1 Proyecto Interno Antecedentes para la historia de las Ciencias Naturales en la región de Magallanes.

2 Laboratorio de Entomología, Instituto de la Patagonia, Universidad de Magallanes. Casilla 113-D. Punta Arenas, Chile. vicente. perez@umag.cl 
Charles Darwin (12-2-1809 a 19-4-1882), gran observador de la naturaleza y apasionado recolector principalmente de insectos, tuvo el privilegio de participar en una de las más largas exploraciones científicas del siglo XIX y no pudo escapar a la creación de epónimos que lo han inmortalizado, aunque posiblemente por su gran modestia, sentía antipatía por la práctica de los taxónomos que contribuía a la perpetuación de ciertas personas en el tiempo ( $\mathrm{F}$. Darwin 1887, fide Smith 1987), lo que podría estar relacionado con la superlativa adulación de su nombre en la formación de tantos epónimos (Smith 1987).

En la expedición inglesa 1831-1836 del HMS. Beagle, corbeta bergantín con apenas veintisiete metros y medio de eslora, 235 toneladas de desplazamiento, diez cañones, con capacidad para 74 hombres, al mando del capitán de 24 años Robert FitzRoy, hidrógrafo y meteorólogo, iba Charles Robert Darwin, de 21 años, como naturalista no oficial y como compañero de mesa en el camarote del capitán (sólo en la noche, ya que durante el día compartía el rancho con los oficiales).

La misión del Beagle era completar el levantamiento de mapas de la costa de Patagonia y Tierra del Fuego, iniciado durante su viaje anterior (1826-1830), al mando del capitán Pringle Stokes, y dar la vuelta al mundo.

El médico Robert Waring Darwin, padre de Charles, pagó los gastos de su hijo, compró el equipo que necesitaba para desempeñarse y hasta le proporcionó un ayudante.

En un minúsculo camarote en la popa del barco, Charles Darwin, con su ayudante Syms Covington, preparaba las toneladas de especímenes de historia natural que recolectaban en los lugares en que recalaba el Beagle y que eran enviados a Inglaterra, donde los recibía el reverendo John Steven Henslow, ex profesor de y tutor informal de Darwin en el St. John's College de Cambridge, quien tuvo el mérito de haber transformado al precoz coleccionista de escarabajos en un naturalista en ciernes: fue él el encargado de buscar y proponer a un candidato para el cargo tan especial en el Beagle, recomendarlo $e$ instarlo a no perder esa oportunidad. Además, mantuvo correspondencia con Darwin durante los cinco años de la expedición, colaborando a distancia, guiándolo en sus cometidos, y a quien su ex alumno consideraba como el único que no le hizo perder el tiempo en su educación universitaria (Milner 1995).
El 27 de diciembre de 1831, cuando el H.M.S. Beagle partió desde el puerto de Portsmouth, fue considerado por el propio Darwin como su "auténtico nacimiento", y los cinco años de viaje de descubrimiento a borde del buque como el período de su "auténtica educación". En su autobiografía, escribió que a la edad de ocho años el gusto que sentía por la historia natural cuando empezó a asistir al colegio de Shrewsbury, se había convertido en una pasión por la recolección de especímenes, que podría conducir a un hombre a ser un naturalista sistemático, un virtuoso o un miserable (Porter 1987).

El viaje en el Beagle se constituyó en una gran oportunidad para su pasión de formar colecciones. Aunque sus principales intereses en el viaje del Beagle fueron la Geología y la Zoología ("La Geología y los animales invertebrados serán mi principal objetivo durante todo el viaje" expresa en carta a su mentor en Botánica el Reverendo John Stevens Henslow en la Universidad de Cambridge), Darwin recolectó plantas y también hizo observaciones sobre la vegetación; por su parte, Henslow comprendía que como Darwin no era un botánico, las herborizaciones que realizaba eran para agradarlo a él (Porter 1987).

A Jackson (1909) se debe una lista de 44 especies de vegetales dedicados a Charles, procedentes de distintos países (incluyendo una fósil, de Rusia), 4 de Chile, 12 de Patagonia y 5 de la Región Magallánica; R.B. Freeman (1915-1966) recopiló unos 200 nombres de lugares, vegetales y animales bautizados con el nombre de Darwin (fide Milner 1995); según Milner (1995) alrededor de 100 seres vivos llevan el nombre de Darwin.

Los insectos, que despertaron la pasión del recolector Charles Darwin a los diez años de edad (F. Darwin 1887, fide Smith 1987), y que el gran naturalista capturaba, ordenaba y despachaba a Inglaterra con la colaboración de su ayudante Syms Covington, cada vez que el Beagle recalaba en algún puerto, fueron presentados por Smith (1987) con indicación de sus repositorios: British Museum (Natural History); University Museum of Zoology, Cambridge; Hope Entomological Collections, University Museum, Oxford; y National Museum of Ireland, Dublin; en Downw House, la casa de Darwin, con una sola excepción, no hay insectos del viaje en el Beagle. También se incluye una lista de 132 epónimos de insectos. 
El objetivo de este artículo es recordar al gran naturalista Charles Darwin a 200 años de su nacimiento y de cuyo paso por Chile, 1 año y 93 días, según el itinerario publicado por Barlow (1967, fide Smith 1987) dan fe, aunque mínimamente, los epónimos erigidos en su honor referidos a áreas geográficas que visitó o especies dedicadas a él basadas en material que él recolectó en nuestro territorio.

\section{MATERIAL Y MÉTODOS}

Para ubicar los epónimos topónimos, u homónimos, según la denominación de los autores, se consultaron diccionarios geográficos, atlas y listas de nombres geográficos. Para los nombres específicos y epítetos específicos relacionados con Darwin, se revisaron catálogos estrictamente taxonómicos, monografías y tratados en una primera aproximación; posteriormente, se continuó la búsqueda para determinar qué especies habían sido descritas sobre material recolectado por Darwin en Chile.

\section{RESULTADOS}

GEA

Geografía

Con el nombre Darwin fue bautizada una decena de accidentes geográficos (bahías, bancos, canales, cerros, cordilleras, ensenadas, estrechos, islas, montes, picos, senos, ventisqueros), casi todos situados entre los paralelos 45 y 55 de latitud sur, excepto una cordillera en el paralelo 27 (AstaBuruaga 1899, Riso Patrón 1924, U.S.A. Board on Geographic Names 1967, Instituto Geográfico Militar 1974, Mantellero 2000).

\section{Geología}

A Darwin se le reconoce como el iniciador del estudio de las terrazas marinas de la Patagonia; es el primer autor que se refirió a los depósitos de rodados ("gravel formations") de las mesetas patagónicas; precedió a los demás autores (Grange, Steinmann, Felsch, Bonarelli, Keidel, Hemmer, Decat y Pomeyrol) en el estudio de los terrenos precretácicos de la región magallánica; fue el primero en señalar la existencia de depósitos glaciares de la Patagonia y zona del estrecho de Magallanes (Feruglio 1949).
Mineralogía

Forbes (1860) describió la especie mineral darwinita, basado en una muestra que llegó a sus manos calificada como de arsénico y constituida por $88,37 \%$ de cobre y $11,63 \%$ de arsénico, a la que nominó "en honor de Darwin, cuyo admirable examen geológico de esta parte de Sudamérica es tan conocido que no requiere mayor comentario". Provenía de una localidad cercana a Potrero Grande "no muchas millas al sudeste de la ciudad de Copiapó en el norte de Chile". Probablemente se trate de la mina homónima de cobre (30³0'S; 71²0'W) (Riso Patrón 1924, obra que registra tres topónimos homónimos más en la misma zona). Page (1865) la registra para Chile y Perú, destacando que fue nominada en homenaje a Darwin "por sus investigaciones geológicas en Sudamérica".

Sobre el recolector, no hay noticias. De la localidad Copiapó, sí se sabe que el Beagle estuvo allí, de donde zarpó el 6 de julio de 1835 (Barlow 1967, fide Smith 1987). Según el ingeniero de minas De Novo y F. Chicarro (1957), darwinita "es un nombre antiguo de la whitneyita (mineral dedicado a J.D. Whitney, geólogo norteamericano), con yacimientos en Michigan, California, Sonora (México) y Copiapó; Mikhail et al. (2009) registran whitneyita para México, pero no mencionan darwinita; sin embargo, en la bibliografía mineralógica histórica no se registra darwinita ni whitneyita para Chile (Brüggen 1931). Por no saber cómo operan los mineralogistas en estos casos de posibles sinonimias, y no haber podido obtener una respuesta aclaratoria y definitiva a las consultas que se han hecho, sólo se consigna aquí la creación del nombre darwinita.

FLORA (Hooker \& Arnott 1841, Marticorena \& Quezada 1985, Moore 1983, Stuardo 1960).

Pteridophyta

Hymenophyllum darwinii Hook. f. ex V. den Bosch (Hymenophyllacea). Tierra del Fuego.

Angiospermae

Berberis darwinii Hook 1844 (Berberidaceae). Chiloé.

Nassauvia darwinii (Hooker \& Arn. 1836) O. Hoffm. et Dusén 1901 (Compositae). Port Gregory.

Senecio darwinii Hooker \& Arn. 1841 (Compositae). Parte sur de Tierra del Fuego. 
Carex darwinii Boott. var. darwinii (Cyperaceae). Región Magallánica.

Poa darwiniana Parodi 1937 (= Triodia antarctica Hooker f. 1847) (Gramineae). Tierra del Fuego.

El escaso número de ejemplares de especies de plantas nuevas para la ciencia recolectados por Darwin en Chile, y dedicadas a él, puede deberse a tres causas: a) la recolección de plantas vasculares se inició muy temprano (G. Handisyd ya había herborizado en 1690 en isla Isabel, fide Moore 1983), b) las recaladas del Beagle en Sudamérica iban sucediéndose desde latitudes bajas (Bahía, en Brasil) hasta culminar en latitudes tan altas como las del estrecho de Magallanes, con las consiguientes descripciones anteriores de especies), y c) las herborizaciones de Darwin fueron costeras, por las variables condiciones del clima (Moore 1983).

FAUNA (Stuardo 1960)

ARTHROPODA

INSECTA (Smith 1987)

Delphacodes darwini Muir, 1929 (Hemiptera).

Isla de Chiloé.

Melizoderes darwini Funkhouser, 1934

(Hemiptera). Isla de Chiloé.

Aulonodera darwini Champion, 1918 (Coleoptera). Isla de Chiloé.

Carabus darwinii Hope, 1838 (Coleoptera). Isla de Chiloé.

Migadops darwini G.R. Waterhouse, 1842 (Coleoptera). Isla Navarino, Tierra del Fuego.

Orynipus darwini Brèthes, 1924 (Coleoptera). Isla de Chiloé.

Parahelops darwini C.O. Waterhouse, 1875 (Coleoptera). Tierra del Fuego y Valparaíso.

Polylobus darwini Bernhauer, 1935 (Coleoptera). Isla de Chiloé.

Stictospilus darwini Brèthes, 1924 (Coleoptera). Chile.

Leptocera darwini Richards, 1931 (Diptera) Concepción.

Pelecorhynchus darwini Ricardo, 1900 (Diptera). Chiloé.

Pelycops darwini Aldrich, 1934 (Diptera). Tierra del Fuego.

Valdivia darwini (Shannon, 1927) (Diptera) (=Valdiviomyia). Chile.

\section{CHORDATA}

MAMMALIA (Muñoz-Pedreros 2000, Tamayo \& Frassinetti 1980).

Phyllotis darwini (Waterhouse, 1837) (Rodentia). Coquimbo.

AVES (Philippi 1964)

Traupis bonariensis darwini (Bonaparte, 1837). Chile.

AMPHIBIA (Cei 1962)

Rhinoderma darwini Duméril \& Bibron, 1841 (Amphibia). Valdivia.

\section{AGRADECIMIENTOS}

Expresamos nuestros agradecimientos a las siguientes personas que gentilmente atendieron nuestras consultas: geólogo Ernesto Pérez, ingeniero en minas Tihomir Domic, botánicas Melica Muñoz y Elizabeth Barrera.

\section{LITERATURA CITADA}

Asta-Buruaga, F.S. 1899. Diccionario Geográfico de la República de Chile. NuevaYork. Appleton y Compañía. 903 pp.

Brüggen, J. 1931. Tablas para la determinación de los minerales. Establecimientos Gráficos "Barcelles \& Co." Santiago de Chile. 204 pp.

Cei, J.M. 1962. Batracios de Chile. Ediciones de la Universidad de Chile. Santiago de Chile. 128 pp. + láminas y apéndice: i-cviii.

De Novo, P. \& F. Chicharro 1957. Diccionario de Geología y Ciencias Afines. Tomo I. Geografía Física, Cristalografía, Mineralogía, Petrografía. Editorial Labor, S.A. 796 pp.

Feruglio, E. 1949. Descripción geológica de la Patagonia. Tomos I y II. Buenos Aires. Imprenta y Casa Editora "Coni".

Forbes, D. 1860. On Darwinite: A New Mineral Species from Chile. The London, Edinburgh and Dublin Philosophical Magazine and Journal of Science Fourth Series, 20:423-426.

Hooker, W.J. \& G.A.W. Arnott 1836. Contributions towards a flora of South America and the islands of the Pacific. I. Extra-Tropical South America. Companion to the Botanical Magazine 2:41-52. Online: http://darwin-online. org.uk/content/frameset? itemID $=\mathrm{A} 152 \&$ viewtype=image \& pageseq $=1$ 4/4/2009. 
Instituto Geográfico Militar 1970. Atlas de la República de Chile. Instituto Geográfico Militar. 249 pp.

Jackson, D. 1909. Plants named after Darwin. In Darwin Centenary Number, pp. 242-244. Christ's College Magazine, Easter Term, Cambridge, 23(70):183-225.

Mantellero, C.A. 2000. Diccionario Geográfico Náutico. Litografía Carroza, S.A. Valparaíso. $261 \mathrm{pp}$.

Marticorena, C. \& M. Quezada 1985. Catálogo de la flora vascular de Chile. Gayana 42(1-2):1-157.

Mikhail, O., P. Corona, J. Díaz de León, A. Victoria \& Cruz 2009. Taxonomía y Clasificación Cristaloquímica de los Minerales Mexicanos. Online: http://www.geocities.com/ smexmineralogia/catalogo.htm 30/3/2009.

Milner, R. 1995. Diccionario de la evolución. Biblograf S.A. Impreso en HEDIM, S.C.C.L. Barcelona, 685 pp.

Moore, D.M. 1983. Flora of Tierra del Fuego. Anthony Nelson. England. Missouri Garden, USA. 396 pp.

Muñoz-Pedreros, A. 2000. Orden Rodentia. In A. Muñoz-Pedreros \& J. Yáñez (Eds.), Mamíferos de Chile pp. 73-126. Ediciones Cea. Impreso en Chile.

Page, D. 1865. Handbook of Geological Terms, Geology and Physical Geography. William Blackwood and Sons. Edinburgh and London. Second Edition, Revised and enlarged.
Online: http://books.google.cl/books?id=c Bw5AAAAMAAJ\&dq=David+Page $:+$ Hand book+ of+Geological+Terms, + G.

Philippi, R.A. 1964. Catálogo de las Aves Chilenas con su distribución geográfica. Investigaciones Zoológicas Chilenas 11:1-179.

Porter, D.M. (Ed.). 1987. Darwin's notes on Beagle plants. Bulletin of the British Museum (Natural History) Historical Series 14(2):145-233.

Riso Patrón, L. 1924. Diccionario Jeográfico de Chile. Imprenta Universitaria. Santiago. 599 pp.

Smith, K.G.V. (Ed). 1987. Darwin's Insects. Charles Darwin's Entomological Notes. Bulletin of the British Museum (Natural History), Historical Series, 14(1):1-143.

Stuardo, C. 1960. Historia Física y Política de Chile de don Claudio Gay. Índices alfabéticos de la Botánica y de la Zoología. Publicaciones del Centro de Estudios Entomológicos, Facultad de Filosofía y Educación, Universidad de Chile, 3.

Tamayo, M. \& D. Frassinetti 1980. Catálogo de los mamíferos fósiles y vivientes de Chile. Boletín del Museo Nacional de Historia Natural 37:323-399.

U.S.A. Board on Geographic Names 1967. Chile. Second Edition. Official Standard Names approved by the United States Board on Geographic Names. Prepared by the Office of Geography, Department of the Interior, Washington, D.C. 
